\title{
Fifty Star Cluster Candidates toward the Galactic Bulge from VVV and Gaia
}

Dante Minniti $^{1,2,3}$, Javier Alonso-García ${ }^{2,4}$, Jura Borissova ${ }^{2,5}$, Juan José Clariá ${ }^{6}$, Bruno Dias $^{1,2}$, Matias Gomez ${ }^{1}$, Maren Hempel ${ }^{1}$, Radostin Kurtev ${ }^{2,5}$, Tali Palma ${ }^{6}$, Joyce B.

Pullen ${ }^{2}$ Show full author list

Published 2019 July 23 • (C) 2019. The American Astronomical Society. All rights reserved. Research Notes of the AAS, Volume 3 , Number 7

$\underline{\text { References }}$

64 Total downloads

Get permission to re-use this article

Share this article

$\underline{\text { Article information }}$

Export citation and abstract $\underline{\text { BibTeX }} \underline{\text { RIS }}$

Globular clusters (GCs) are the oldest and most massive star clusters in the Galaxy, and are preferentially destroyed in the central bulge. Most of the dynamical processes that affect GC survival (bulge shocking, dynamical friction, tidal disruption, evaporation, etc.) are maximized in the Galactic bulge. That is why the bulge has been called the elephant graveyard of the Milky Way (Minniti et al. 2017a). It is in the bulge where we expect to find GCs in different stages of evolution: normal GCs, clusters in process of disruption, as well as the debris of already destroyed GCs.

Searching for these objects in the innermost regions of the Milky Way is very difficult due to the high foreground and background contamination, and to the extreme extinction and crowding. Large surveys like 2MASS (Skrutskie et al. 2006), Glimpse (Benjamin et al. 2005), VVV (Minniti et al. 2010), and Gaia DR2 (Gaia Collaboration et al. 2018), have recently allowed the discovery of numerous new GCs in these inner regions. These newly found GCs are mostly faint and small, although a couple of large ones have also been 
identified lately (like VVV-GC05-Minniti et al. 2017d, and FSR1758 - Cantat-Gaudin 2018; Barba et al. 2019). The recent compilation of 10,000 star clusters by Bica et al. (2019) comprises the most complete and up to date list, including a number of new GC discoveries by different teams (e.g., Froebrich et al. 2007; Borissova et al. 2014; Minniti et al. 2017a, 2017b, 2017c; Camargo 2018; Ryu \& Lee 2018, etc.).

Herewith we have extended our previous bulge GC searches following the procedures described by Minniti et al. (2017a, 2017b, 2017c), adding a couple of additional steps to target more distant objects. Considering the high foreground contamination and in order to discard nearby sources, we used the matched VVV-Gaia catalogs of Kammers et al. (2019),

with distances (Bailer Jones et al. 2018). Also, we rejected sources with proper motions consistent with the mean motion of the Galactic disk for the different fields. We then built red giant density maps and identified the over-densities with sizes typical for GCs. Finally, we checked the optical and near-IR color-magnitude diagrams for each individual object, selecting the ones with red giant branches tighter than their surrounding fields. While in most targets a clear red clump is present, in a few cases there is also a concentration of RR Lyrae stars and/or a blue horizontal branch can be observed.

The fifty new candidate GCs are listed in Table $\underline{1}$. We give their IDs, positions, mean field extinction values $A_{K s}$ (Schlafly \& Finkbeiner 2011), and the number of RR Lyrae within 3 arcmin of the cluster center. As a caveat, we cannot discard the possibility that some of these GC candidates could be open clusters or mere background fluctuations instead of real GCs. These objects must be individually confirmed as such using additional information (like proper motions, radial velocities, chemical abundances, presence of RR Lyrae variable stars, etc.), as discussed for example by Minniti et al. (2018), and Palma et al. (2019). These new GC candidates are interesting cases for a variety of follow-up studies.

Table 1. New VVV-Gaia GC Candidates in the Galactic Bulge

\begin{tabular}{lcllll} 
ID & R.A.(J2000)Decl.(J2000) & \multicolumn{3}{c}{$N_{R R L}$ Comment $^{\mathrm{a}}$} \\
Minni 85 & $18: 02: 25$ & $-28: 43: 52$ & 0.233 & 1 & \\
Minni 86 & $18: 15: 26$ & $-30: 15: 56$ & 0.073 & 1 & \\
Minni 87 & $18: 13: 48$ & $-27: 57: 23$ & 0.141 & 4 & BHB \\
Minni 88 & $18: 21: 10$ & $-28: 14: 23$ & 0.113 & 1 & \\
Minni 89 & $17: 47: 59$ & $-21: 24: 34$ & 0.301 & 0 & \\
Minni 90 & $18: 16: 46$ & $-34: 19: 41$ & 0.050 & 0 & BHB \\
Minni 91 & $17: 54: 14$ & $-36: 09: 08$ & 0.172 & 3 & BHB \\
Minni 92 & $18: 01: 04$ & $-36: 58: 28$ & 0.086 & 0 & BHB \\
Minni 93 & $18: 09: 04$ & $-37: 50: 36$ & 0.041 & 0 & \\
Minni 94 & $17: 15: 03$ & $-31: 48: 46$ & 0.314 & 1 & \\
Minni 95 & $18: 14: 22$ & $-21: 53: 04$ & 0.728 & 1 & \\
Minni 96 & $18: 14: 10$ & $-21: 39: 17$ & 0.951 & 0 & \\
Minni 97 & $18: 12: 34$ & $-20: 49: 01$ & 1.155 & 0 & \\
Minni 98 & $18: 10: 45$ & $-22: 32: 20$ & 0.953 & 0 & \\
Minni 99 & $18: 14: 35$ & $-22: 01: 11$ & 0.704 & 1 &
\end{tabular}




$\begin{array}{lllll}\text { ID } & \text { R.A.(J2000)Decl.(J2000) } & N_{R R} \\ & & & & \\ \text { Minni 100 } & 18: 08: 58 & -23: 47: 08 & 1.113 & 1 \\ \text { Minni 101 } & 17: 34: 56 & -33: 37: 54 & 2.116 & 2 \\ \text { Minni 102 } & 17: 24: 04 & -36: 14: 54 & 3.087 & 0 \\ \text { Minni 103 } & 17: 27: 41 & -37: 50: 40 & 0.869 & 0 \\ \text { Minni 104 } & 17: 32: 18 & -37: 58: 18 & 0.482 & 1 \\ \text { Minni 105 } & 17: 28: 56 & -37: 41: 25 & 1.049 & 0 \\ \text { Minni 106 } & 17: 32: 59 & -36: 44: 30 & 0.454 & 1 \\ \text { Minni 107 } & 17: 34: 26 & -37: 01: 29 & 0.484 & 0 \\ \text { Minni 108 } & 17: 31: 03 & -37: 17: 26 & 0.798 & 0 \\ \text { Minni 109 } & 17: 29: 08 & -38: 17: 56 & 0.689 & 0 \\ \text { Minni 110 } & 17: 32: 45 & -37: 41: 21 & 0.571 & 0 \\ \text { Minni 111 } & 17: 31: 41 & -37: 49: 38 & 0.587 & 0 \\ \text { Minni 112 } & 17: 32: 24 & -36: 20: 43 & 0.650 & 2 \\ \text { Minni 113 } & 17: 35: 34 & -35: 50: 08 & 0.709 & 0 \\ \text { Minni 114 } & 17: 38: 07 & -35: 44: 44 & 0.460 & 1 \\ \text { Minni 115 } & 17: 37: 11 & -34: 35: 37 & 0.688 & 1 \\ \text { Minni 116 } & 17: 34: 54 & -33: 38: 24 & 2.128 & 2 \\ \text { Minni 117 } & 17: 35: 34 & -34: 55: 39 & 0.807 & 0 \\ \text { Minni 118 } & 17: 41: 00 & -34: 51: 51 & 0.533 & 0 \\ \text { Minni 119 } & 17: 34: 58 & -33: 39: 33 & 2.145 & 0 \\ \text { Minni 120 } & 17: 18: 59 & -34: 02: 42 & 0.778 & 0 \\ \text { Minni 121 } & 17: 17: 53 & -34: 26: 09 & 0.767 & 0 \\ \text { Minni 122 } & 17: 33: 31 & -36: 57: 19 & 0.454 & 1 \\ \text { Minni 123 } & 17: 38: 10 & -34: 51: 09 & 0.622 & 0 \\ \text { Minni 124 } & 18: 05: 06 & -25: 17: 25 & 1.048 & 2 \\ \text { Minni 125 } & 18: 08: 37 & -24: 34: 19 & 0.666 & 1 \\ \text { Minni 126 } & 18: 10: 22 & -23: 14: 38 & 0.736 & 1 \\ \text { Minni 127 } & 18: 08: 05 & -23: 49: 27 & 1.104 & 1 \\ \text { Minni 128 } & 17: 25: 34 & -38: 31: 40 & 0.607 & 0 \\ \text { Minni 129 } & 17: 09: 06 & -37: 40: 42 & 0.946 & 0 \\ \text { Minni 130 } & 17: 20: 53 & -40: 02: 01 & 0.546 & 0 \\ \text { Minni 131 } & 17: 16: 50 & -40: 00: 34 & 0.852 & 0 \\ \text { Minni 132 } & 16: 59: 22 & -39: 11: 31 & 0.829 & 0 \\ \text { Minni 133 } & 17: 08: 13 & -42: 51: 00 & 0.896 & 0 \\ \text { Minni 134 } & 16: 46: 41 & -42: 40: 06 & 0.929 & 0 \\ \text { Minni 135 } & 15: 40: 36 & -56: 22: 45 & 1.345 & 0\end{array}$

Note.

${ }^{\mathrm{a}} \mathrm{BHB}$ indicates the possible presence of a blue horizontal branch.

\section{References}

- Bailer-Jones C. A. L. et al 2018 AJ 15658 
$\underline{\text { IOPscienceADSGoogle Scholar }}$

- Barba R. et al 2019 ApJ 870L 24B

$\underline{\text { IOPscienceADSGoogle Scholar }}$

- Benjamin R. A. et al 2005 ApJL 630 L149

$\underline{\text { IOPscienceADSGoogle Scholar }}$

- Bica E. et al 2019 AJ 157 12B

$\underline{\text { IOPscienceADSGoogle Scholar }}$

- Borissova et al 2014 A\&A $56924 B$

$\underline{\text { CrossrefADSGoogle Scholar }}$

- $\quad$ Camargo D. 2018 ApJL 86027

$\underline{\text { IOPscienceADSGoogle Scholar }}$

- Cantat-Gaudin T. 2018 A\&A 618A 93C

$\underline{\text { CrossrefGoogle Scholar }}$

- Froebrich D. et al 2007 MNRAS 374399

CrossrefADSGoogle Scholar

- Gaia Collaboration et al. 2018 A\&A 616 A10

$\underline{\text { CrossrefADSGoogle Scholar }}$

- Kammers R. et al 2019 A\&A submitted

$\underline{\text { Google Scholar }}$

- Minniti D. et al 2010 NewA 15433

CrossrefADSGoogle Scholar

- Minniti D. et al 2017a RNAAS 1 16M

$\underline{\text { ADSGoogle Scholar }}$ 
- Minniti D. et al 2017b RNAAS $154 M$

ADSGoogle Scholar

- Minniti D. et al 2017c ApJL 849 L24

$\underline{\text { IOPscienceADSGoogle Scholar }}$

- Minniti D. et al 2017d ApJL 838 L14

$\underline{\text { IOPscienceADSGoogle Scholar }}$

- Minniti D. et al 2018 ApJ $86612 M$

IOPscienceADSGoogle Scholar

- Palma T. et al 2019 MNRAS in press (arXiv:1905.11835)

$\underline{\text { ADSPreprintGoogle Scholar }}$

- Ryu J. and Lee M. G. 2018 ApJ 86338

$\underline{\text { IOPscienceADSGoogle Scholar }}$

- Schlafly E. F. and Finkbeiner D. P. 2011 ApJ 737 103S

$\underline{\text { IOPscienceADSGoogle Scholar }}$

- Skrutskie M. F. et al 2006 AJ 1311163

$\underline{\text { IOPscienceADSGoogle Scholar }}$

Export references: $\underline{B i b T e X} \underline{\text { RIS }}$

- $\underline{\text { References }}$

Back to top

IOP Science home

- Journals

- Books

- $\quad$ About IOPscience

- Contact us

- Developing countries access

- IOP Publishing open access policy

IOP Publishing home 
- $\quad$ C Copyright 2019 IOP Publishing

- Terms \& conditions

- Disclaimer

- Privacy \& cookie policy This link opens in a new tab.

- This site uses cookies. By continuing to use this site you agree to our use of cookies. 